\title{
COUPLING AGENT EFFECT ON THE TENSILE STRENGTH PROPERTIES OF SISAL FIBER-PLASTIC COMPOSITES
}

\author{
Sigit Baktya Prabawa ${ }^{1}$
}

\begin{abstract}
The fiber-plastic composites were made from the compounding of plastic (polypropylene) and sisal (Agave sisalana) fiber with or without addition of coupling agent (Maleic anhydride grafted polypropylene). The tensile strength (Modulus of Elasticity-MOE, Modulus of Rupture-MOR) of the composites were evaluated in the three different conditions, i.e. air dry condition (control), 24 - hour immersion in cold water and 2 -hour immersion in boiling water. The results showed that the addition of coupling agent enhanced the properties of the composites. These properties did not significantly change although the composites were immersed in cold water as long as 24 hours. However, immersion in boiling water for 2 hours, tended to decrease MOR and MOE of the composites.
\end{abstract}

Keywords: plastic, sisal, composite, tensile strength, coupling agent

\section{INTRODUCTION}

Sisal (Agave sisalana) fiber can be grouped as lignocellulosic fiber stuffs. Generally, these stuffs are any substances that contain mainly both holocellulose and lignin, and include materials such as wood, agricultural residues, water plants, grasses and other plant substances (Rowell and Young, 1989) or any parts of plants such as stems, straws, stalks, basts, barks, leaves, seed hairs and so on. When commuted into particles or fibers, they can provide raw materials for a broad array of products. Therefore, lignocellulosic fiber-plastic composites can be defined as composites where the lignocellulosic fibers are compounded with plastics, but each of these two components still remains as a distinct separate phase (Youngquist and Rowell, 1989).

Some advantages of lignocellulosic fiber-plastic are affording low cost, diversity in applications, and use of native lignocellulosic raw materials. A list of potential products of the fiber-plastic composites includes (Sanadi et al., 1994): storage bins for crops or other commodities, garbage cans, packaging applications comprising containers, cartons and pallets, sporting goods, fence posts, automobile and truck components both for exterior and interior parts, paneling for interior wall sections, partitions and door systems, furniture components including both flat and curved surfaces, temporary housing structure, floor, wall and roof systems for light-frame construction as well as recreational materials.

When lignocellulosic fibers such as sisal, kenaf, ramie, abaca fibers are mixed with thermoplastic compounds such as polyethylene or polypropylene (PP) logically, there occur some constraints due to the differences in surface properties. The difficulties in compounding these two materials and the poor efficiencies in stress transfer are because of

\footnotetext{
${ }^{1}$ Forest Research and Development of Kalimantan, Samarinda
} 
the intrinsic polar or hydrophilic nature of the lignocellulosic fibers and the hydrophobic or non-polar characteristics of the polyolefins (Sanadi and Caulfield, 1994). Some efforts have been made to remedy this limitation by applying conventional coupling agents, but these are still either too costly or unable to work efficiently in lignocellulosic-thermoplastic systems (Sanadi and Caulfield, 1994). However, recent work has shown that the use of maleic anhydride-grafted polypropylene can improved properties of the resulting composites. Compatibility and stress transfer in the composites depended on the amount of maleic anhydride introduced into the graft copolymer and the molecular weight of the copolymer as has been experienced with recycled newspaper fibers-polypropylene composite systems (Tai, 1993).

Intrinsically, the mechanical and physical properties of lignocellulosic fiber-plastic composites are governed by the properties and amount of all materials incorporated in the system, such as resins or thermoplastic agents, coupling agents or additives and lignocellulosic fibers acted as fillers and/or reinforcements, and also by processing techniques.

Fillers have played an important role in the plastic industry because they reduce cost and/or enhance certain properties, such as stiffness and thermal properties, of the final plastic products (Sanadi and Caulfield, 1994). The use of specieal fillers such as glass fibers incorporated into the plastics to improve certain properties generally results in higher cost than typical fillers such as $\mathrm{CaCO}_{3}$ or talc (Sanadi and Caulfield, 1994). The fillers or fibers, used in the plastics can absorb moisture and affect the properties of the composites. This effect depends on the type of exposure (by immersion in water or exposure to high humidity), the shape of the component, the inherent properties of the plastic, and the quality of the fiber-matrix interface (Sanadi and Caulfield, 1994).

The aim of this study was to evaluate the tensile strength properties of lignocellulosic fiber-plastic composites prepared from sisal (Agave sisalana) fibers and polypropylene, especially to understand better the effect of coupling agents addition on the tensile properties of sisal fiber-plastic composites.

\section{MATERIALS AND METHODS}

\section{A. Materials}

The main materials used in this study were lignocellulosic fiber, plastic and coupling agent. In this experiment, the lignocellulosic fibers derived from sisal fibers (A. sisalana Perrine) were obtained from leaves of sisal plants grown in Blitar, Jawa Timur. The plastic used was polypropylene homopolymer under the trade mark of Fortilene PP 1620 bought from Solvay Polymers, Inc., TX, in the pellet form. Meanwhile the coupling agent used was maleic anhydride grafted polypropylene called Epolene G-3002 from Eastman Chemical Co., $\mathrm{TN}$, in the pellet form.

\section{B. Method of Making Composite}

By using a simple machine, sisal fibers were extracted from the fresh leaves and then immediately washed with water to remove non-fiber residuals. The fibers subsequently were spread out and dried in the sun. The sisal fibers called fiber strands were cut to about one third the original length and then chopped again to obtain maximum fiber lengths of about $1 \mathrm{~cm}$. 
Sisal fibers, polypropylene, with or without modification with coupling agent, were blended for some particular duration in a high-intensity kinetic mixer (Synergistics Industries Ltd., Canada) where the only heat source was from the kinetic energy of the rotating blades. The blade rotated at $4600 \mathrm{rpm}$ to homogenized the blend and each batch of the blade was operated for about 2 minutes. The blend was automatically discharged from the mixer when the temperature reached $190{ }^{\circ} \mathrm{C}$. Each batch in total weight of $150 \mathrm{~g}$, and about $1.8 \mathrm{~kg}$ of blended material was prepared for each experimental set in the batch.

In all experiments, each of the blends contained by weight $50 \%$ of sisal fibers and an equivalent $50 \%$ of the matrix materials (PP and coupling agent). The amount of coupling agent varied from 0 to $4 \%$. The blends were then granulated and dried at $105^{\circ} \mathrm{C}$ for 4 hours to remove moisture. The granulated blends were injected into the molding chest and subsequently molded at about $190^{\circ} \mathrm{C}$ using a Cincinnati Milacron 33 ton vista sentry injection molding machine. The specimen were prepared with sizes in accordance with the respective ASTMD638-90.

\section{Testing Procedure}

Tensile strength (modulus of rupture - MOR and modulus of elasticity - MOE) tests for sisal fiber plastic composites were conducted under the following three different conditions, i.e. dry, after 24-hour immersion in distilled water, and after 2 - hour immersion in boiling distilled water. The testing procedures were conducted according to the ASTM D638-90 for the tensile test.

\section{Experimental Design}

The design of the experiments were to compare the tensile strength (MOR and MOE) of the composites prepared with different percentages of coupling agents. Two-way analysis of variances were employed to analyze the data using significant different level (á) at $5 \%$.

\section{RESULTS AND DISCUSSION}

\section{A. Modulus of Rupture (MOR)}

The tensile strength properties (MOR) of sisal fiber-plastic composites prepared at various different levels of the coupling agents under three different condition, i.e. dry (control), 24-hour cold water immersion and 2-hour boiled water immersion are summarized in Table 1. Since there were tendencies that the higher the means of MOR the higher its standard deviation, the square root transformed MOR data were hence used in analysis of variances.

Figure 1 shows the MOR of sisal fiber plastic composites followed the function of percent coupling agent for sisal fiber-plastic composites under three different conditions (control, 24 hour cold water immersion and 2 hour boiled water treatment). Meanwhile, MOR of sisal fiber plastic composites also conformed to the function of three different conditions. 
Table 1. The averages and standard deviations of the tensile strength properties (MOR) of sisal fiber-plastic composites

\begin{tabular}{|c|c|c|c|c|c|c|c|}
\hline \multirow{2}{*}{$\begin{array}{c}\text { Tensile } \\
\text { Strength }\end{array}$} & \multirow{2}{*}{$\begin{array}{c}\text { Coupling } \\
\text { Agent } \\
\text { Properties }\end{array}$} & \multicolumn{2}{|c|}{\begin{tabular}{c}
\multicolumn{6}{|c|}{ Control } \\
$(\%)$
\end{tabular}} & $\begin{array}{c}\text { 24-Hour cold water } \\
\text { Immersion }\end{array}$ & \multicolumn{2}{|c|}{$\begin{array}{c}\text { 2-Hour Boiled } \\
\text { Water }\end{array}$} \\
\cline { 3 - 8 } & & Average & $\begin{array}{c}\text { Standard } \\
\text { Deviation }\end{array}$ & Average & $\begin{array}{c}\text { Standar } \\
\text { Deviation }\end{array}$ & Average & $\begin{array}{c}\text { Standard } \\
\text { Deviation }\end{array}$ \\
\hline Modulus & 0 & 37.11 & 1.15 & 37.75 & 0.92 & 35.27 & 0.21 \\
Of Rupture & 1 & 64.81 & 1.54 & 64.32 & 1.84 & 60.83 & 0.61 \\
(MOR) & 2 & 65.69 & 1.99 & 65.13 & 0.74 & 61.12 & 1.80 \\
(Mpa) & 4 & 67.16 & 0.64 & 65.56 & 2.48 & 64.23 & 0.94 \\
\hline
\end{tabular}

Note: $1 \mathrm{MPa}=1000000 \mathrm{~Pa}$

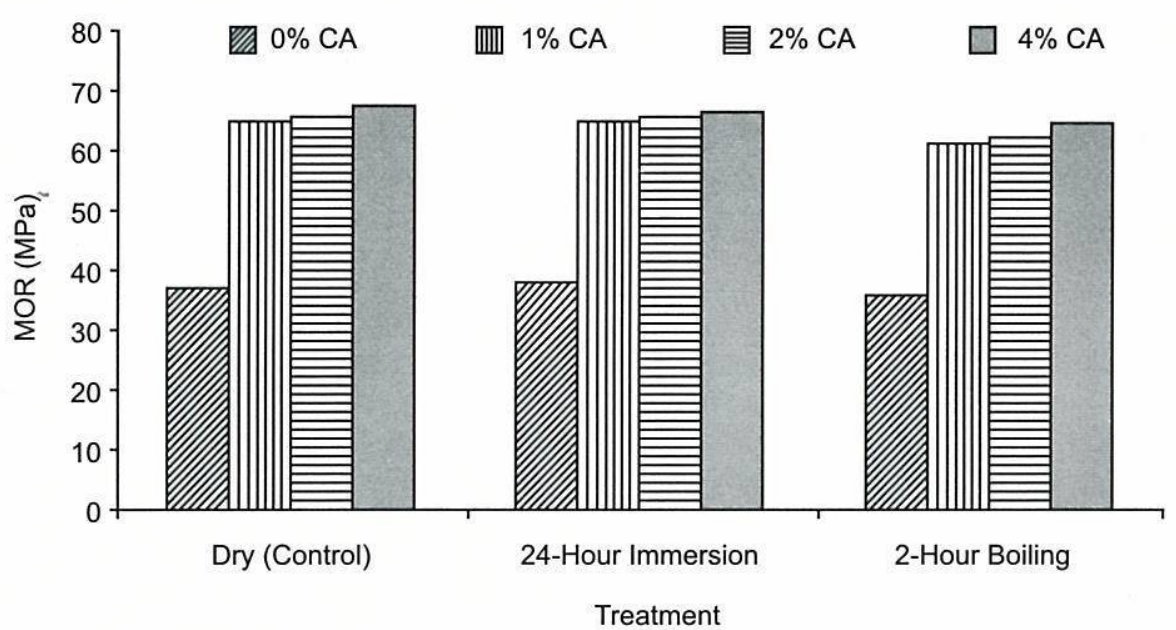

Figure 1. Column chart revealing modulus of rupture (MOR) in relation with percentage of coupling agent for sisal fiber plastic composites

As shown in Figure 1, a small amount of the coupling agent (1\% by weight) improved dramatically the MOR properties of sisal fiber-plastic composites almost twice as much as those of uncoupled ones for all conditions. There were also significant differences in MOR disclosed between the $2 \%$ and $4 \%$ coupling agent addition, but no significant differences between $1 \%$ and $2 \%$ coupling agent. It is believed that the conducive interaction and improved adhesion between the fibers and the matrix, through the formation of covalent bonds, between $\mathrm{OH}$ groups of the fibers and maleic anhydride of the coupling agent, leads to the enhanced stress transfer between the matrix and fibers (Sanadi et al., 1995). The highest MOR $(67.16 \mathrm{MPa})$ was achieved by adding $4 \%$ coupling agent in the sisal fiber plastic 
composites. In quite similar conditions, the tensile strengths (MOR) of sisal fiber plastic composites with $1 \%$ coupling agent $(65 \mathrm{MPa})$ were higher than those of kenaf fiber plastic composites with $1.5 \%$ coupling agent (62 MPa) (Sanadi et al., 1994).

There were no significant difference in MOR between the control (dry) and the 24 hour water immersion test, but there were significant differences between the 24 hour water immersion and the 2-hour boiling water treatment. The former case is probably due to limited penetration of water into the fibers inside the composite matrix during the 24-hour immersion with cold water, thus the absorption of water is not sufficient to plasticize the fibers and therefore the MOR values between control and the immersion test are not significantly different.

The significant differences in MOR values for the 2-hour boiled water treatment indicated that this treatment was sufficient for water to penetrate the matrix and plasticize the fibers inside the composites thereby reducing the MOR.

\section{B. Modulus of Elasticity (MOE)}

The tensile strength properties (MOE) of sisal fiber-plastic composites was prepared at various different levels of the coupling agents and under three different conditions, i.e. dry (control), 24-hour cold water immersion and 2-hour boiling water conditions are summarized in Table 2. Since there were tendencies that the higher the means of MOE the higher its standard deviation, logarithm-transformed MOE data were then used in analysis of variances.

Table 2. The values and standard deviations of the tensile strength properties (MOE) of sisal fiber-plastic composites

\begin{tabular}{|c|c|c|c|c|c|c|c|}
\hline \multirow{2}{*}{$\begin{array}{c}\text { Tensile } \\
\text { Strength }\end{array}$} & \multirow{2}{*}{$\begin{array}{c}\text { Couplin } \\
\text { Agent } \\
\text { Properties }\end{array}$} & \multicolumn{2}{|c|}{\begin{tabular}{c}
\multicolumn{5}{|c|}{ Control } \\
\cline { 3 - 8 }
\end{tabular}} & & \multicolumn{2}{|c|}{$\begin{array}{c}\text { 24-Hour cold } \\
\text { water Immersion }\end{array}$} & \multicolumn{2}{|c|}{$\begin{array}{c}\text { 2-Hour Boiled } \\
\text { Water Treatment }\end{array}$} \\
\cline { 3 - 8 } & & Average & $\begin{array}{c}\text { Standard } \\
\text { Deviation }\end{array}$ & Average & $\begin{array}{c}\text { Standard } \\
\text { Deviation }\end{array}$ & Average & $\begin{array}{c}\text { Standard } \\
\text { Deviation }\end{array}$ \\
\hline Modulus of & 0 & 5.51 & 0.57 & 5.38 & 0.43 & 4.62 & 0.13 \\
Elasticity & 1 & 5.91 & 0.53 & 6.04 & 0.22 & 5.10 & 0.08 \\
(MOE) & 2 & 6.02 & 0.43 & 5.85 & 0.32 & 5.14 & 0.28 \\
(GPa) & 4 & 5.88 & 0.68 & 5.92 & 1.01 & 5.81 & 0.08 \\
\hline
\end{tabular}

Note: $1 \mathrm{GPa}=1,000,000,000 \mathrm{~Pa}$. 


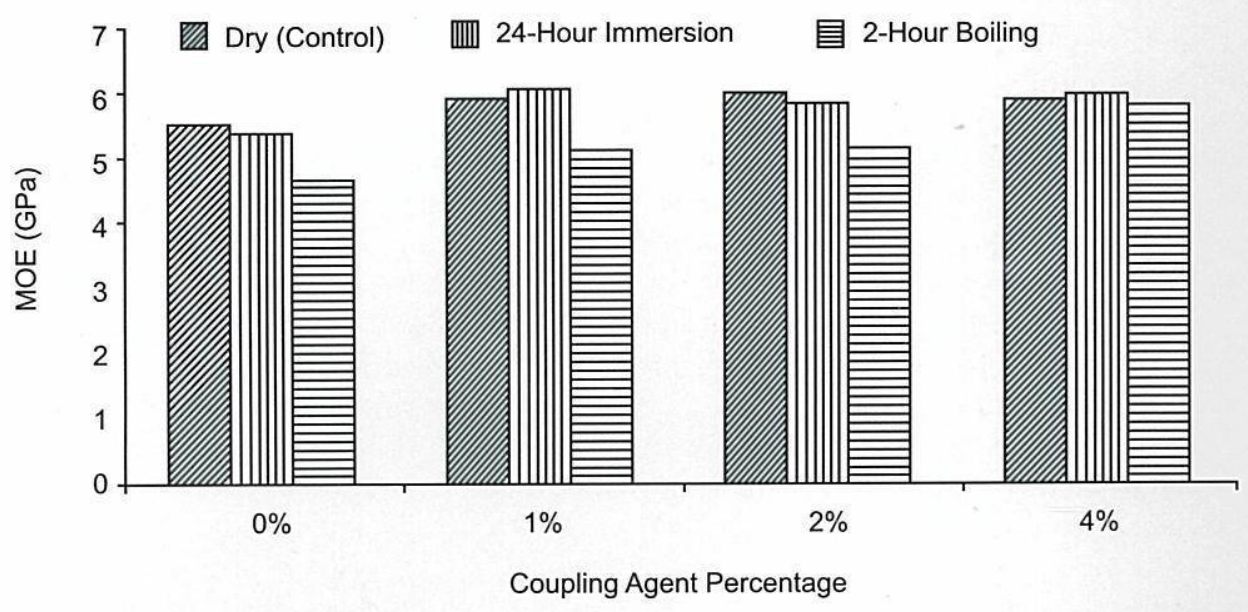

Figure 2. Column chart of modulus of elasticity (MOE) in relation with the percentage of coupling agent for sisal fiber plastic composites

Figure 2 shows the MOE followed the function of changing in percent coupling agent for sisal fiber-plastic composites under three different conditions (control/dry, 24-hour cold water immersion and 2-hour boiling water). Meanwhile, Figure 3 shows the MOE of sisal fiber-plastic composites correspondence to function of different treatment conditions.

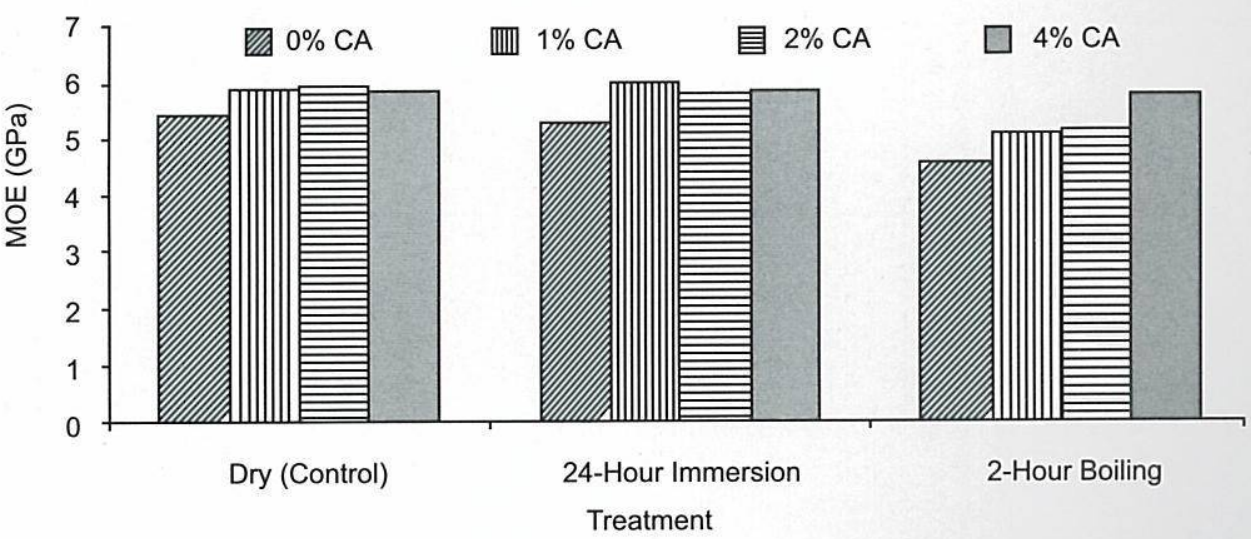

Figure 3. Column chart of modulus of elasticity (MOE) in relation with the different treatments of coupling agents (CA) for sisal fiber plastic composites 
There was a significant difference between $0 \%$ and $1 \%$ coupling agent addition but no significant differences among $1 \%, 2 \%$ and $4 \%$ coupling agent addition (Figure 3). Besides adhesion between the fibers and matrix through covalent bonding induced by coupling agent addition, the improvement of MOE in the composites might be due to the modification of the fiber surfaces caused by coupling agent that prevents formation of transcrystalline regions near the fiber surfaces (Quilin et al., 1993). In this condition elasticities of the fibers would be transferred to the matrix of the composites without which encountered no blocking by transcrystalline zones. Consequently, elastic transfer of unmodified fiber surfaces in composites can be blocked by trancrystalline regions around the fibers thereby lowering the MOE. The MOE of sisal fiber plastic composites with $1 \%$ coupling agent (5.9 $\mathrm{GPa}$ ) was lower than that of kenaf fiber plastic composites, prepared in quite similar condition, with $1.5 \%$ coupling agent $(7.2 \mathrm{GPa}$ ) (Sanadi et al., 1994).

There was no significant difference either in MOE of composites between that of the dry condition (control) and that of 24-hour immersion samples. But, the significant difference in MOE of sisal fiber-plastic composites occured between that of 24-hour cold water immersion and that of 2-hour boiling water treatment (Figure 3). As also happened to the MOR for the case between the control and 24-hour cold water immersion, this similar situation might be due to the low penetration of the water into the fibers in the composite matrix which was not strong enough to plasticize the fibers. On the other hand, the 2-hour boiling water treatment seemed strong enough to plasticize the fibers inside the composites thereby lowering MOR as well on MOE.

\section{CONCLUSIONS AND RECOMMENDATIONS}

\section{A. Conclusions}

1. The tensile strength properties of sisal fiber-polypropylene composites turned out to be affected by the amount of coupling agent added to the composites. Addition of about $1 \%$ maleic anhydride grafted polypropylene (coupling agent) improved the tensile strength properties of the fiber plastic composites. Levels of addition of coupling agent greater than $1 \%$ did not offer substantial improvement in composite properties. It is believed that coupling agent reacts with $\mathrm{OH}$ groups on the fiber surfaces and entangles with the polypropylene matrix.

2. The 24-hour cold water immersion treatment did not affect the tensile strength properties of the sisal fiber-plastic composites. However, the two hour boiling water treatment reduced significantly the tensile strength for both MOR and MOE properties of the sisal fibers-plastic composites.

3. The reduction of the MOR and MOE properties of the composites was due to plasticization of the fibers inside the composites.

\section{B. Recommendations}

1. It is suggested to consider the amount of coupling agent used in this experiment that ranged about 0.25 to $1 \%$ in order to save economically the coupling agent use thereby reducing its cost. 


\section{REFERENCES}

Quillin, D.T., D.F. Caulfield and J.A. Koutsky. 1993. Crystallinity in the polypropylene/cellulose system. I. Nucleation and Crystalline Morphology. J. of Applied Polymer Science, 50:1187-1194

Rowell, R.M. and R.A. Young. 1989. Recent advances in lignicellulosic-derived composites. In Chum, H.L. (Ed.) Assessment of biobased materials. Solar Energy Research Institute, Technical Document - TR 234-3610.

Sanadi, A.R. and D.F. Caulfield. 1994. Development of agricultural fiber filled commodity plastics. Department of Forestry, University of Wisconsin-Madison and Forest Products Laboratory, Madison. Internal report.

Sanadi, A.R, D.F. Caulfield, R.E. Jacobson and R.M. Rowell. 1995. Renewable agricultural fibers as reinforcing fillers in plastics: Mechanical properties of kenaf fiberpolypropylene composites. Ind. Eng. Chem. Res. 34 (5): 1889-1896.

Sanadi, A.R., D.F. Caulfield and R.M. Rowell. 1994. Reinforcing polypropylene with natural fibers. In Plastics Engineering (additives).

Tai, W.C., S.L. Quarles and T.G. Rials. 1993. The effect of compatibilizers on interfacial bonding in lignocellulosic fiber/polyethylene composites. In Cellulosics: chemical, biochemical and material aspects. Kennedy, J.F., G.O. Philips and P.A. Williams (Eds.). Ellis Horwood series in Polymer Science and Technology: 507-512.

Youngquist, J.A. and R.M. Rowell. 1989. Opportunities for combining wood with nonwood materials. In Maloney, T.M. (Ed.) Proceeding of the $23^{\text {rd }}$ international particleboard/ composite materials symposium; 1989 April 4-6; Pullman, WA. Washington State University: 14-157. 